\title{
Dynamic Chunks Distribution Scheme for Multiservice Load Balancing Using Fibonacci Bases Approach
}

\author{
D.Dhivya, \\ Computer Science and Engineering, \\ V.S.B Engineering College, \\ Tamilnadu, India,
}

\author{
U.Gowrisankar \\ Computer Science and Engineering, \\ V.S.B Engineering College, \\ Tamilnadu, India,
}

\begin{abstract}
Cloud computing is collection of distributed hosts which allows services on demand to user. The Centralized cloud based multimedia system CMS[4], materialized because huge number of users demand for various multimedia services through the Internet at the same time and it is hard to design effective load balancing algorithm. Load Balancing is the process which are used to distribute workloads across aggregate computing resources that maximize throughput, minimize latency. In this paper videos are split up into no of chunks and stored at hosts in a distributed manner, The chunk size increased to reduce time lag and improve performance. The cluster heads will monitor all the distribution host loads and client request which could not allow the direct communication between Client and host .Fibonacci-based breaking scheme is introduced to split a video file into number of chunks that allows to reduce the provisioning delay received by users and to optimize the resource utilization by reducing the idle time. The proposed scheme will able to view the whole video by the end user without any delay.
\end{abstract}

Keywords: Cloud computing, load balancing, multimedia system, Fibonacci splitting approach.

\section{INTRODUCTION}

Cloud computing is a model in which the resources are shared by the cloud users via internet. Resources include servers, files, network, applications and services. The important features of cloud computing are broad network access, resource pooling, rapid elasticity, measured service, multi-tenancy. The cloud service providers offer the utilities based on cloud facilities to clients and need to pay for the utilized resources by the time. On demand of number of users for various multimedia computing cloud based multimedia system was emerged [1]. In the proposed system videos are divided into segments that are then further divided into chunks.

These chunks are encoded into independent parts that are distributed to different servers for local storage [2]. The chunk size progressively increased to reduce delay improves performance and reduces the loading time. Since the video data is divided into chunks and stored at peers' local storage in a distributed manner. The cluster heads will monitor all the distribution server loads and client request. This may not allow the direct communication between Client and server and the Server offline problem also managed. Fibonacci-based splitting strategy is introduced to split a video file into number of chunks.

\section{2 .EXISTING SYSTEM}

For optimization genetic algorithm approach was implemented. It is a search algorithm based on the principles of evolution and natural genetics[4]. GA combines the exploitation of past results with the exploration of new areas of the search space. By using survival of the fittest techniques combined with a structured yet randomized information exchange, a GA can mimic some of the innovative flair of a human search.

\section{PROPOSED SYSTEM}

As in proposed describes multimedia system for cloud computing a cluster based multimedia application for the distributed of media files.It addresses how a cloud can perform distributed multimedia processing ,storage and provide quality of service (QoS) provisioning for multimedia services. Different multimedia data dissemination strategies have been analyzed and an innovative technique, based on the Fibonacci series, is proposed .By using this method client can pick up any server without any additional searches.

\subsection{FIBONACCI METHOD}

The Fibonacci sequence is a set of numbers that starts with a one or a zero, followed by a one each number is equal to the sum of the preceding two numbers. If the Fibonacci sequence is denoted as $F(n)$, where $n$ is the first term in the sequence, where the equation follows,

$\mathrm{F}(0)=0,1,1,2,3,5,8,13,21,34$

In mathematical terms, the sequence Fn of Fibonacci numbers is defined by the recurrence relation.

$$
F_{n}=F_{n-1}+F_{n-2}
$$

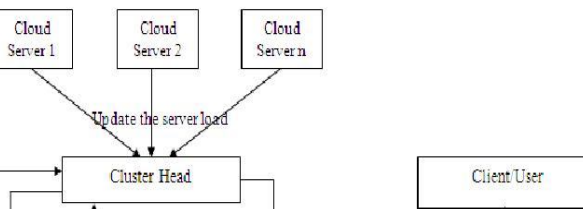




\subsubsection{MULTIMEDIA UPLOAD TO SERVERS}

The Multimedia Upload activity copes with the storage of multimedia contents on the cloud servers. The media files is uploaded in a cloud node and then split (Splitting phase) into number chunks in order to allow the data dissemination and then the parallel tailoring at same time. The number of chunks and the adopted splitting strategies influence the system performances in terms, for example, of delays during the streaming. It is then necessary to carefully carry out the splitting according to the available cloud resources and performing, if possible, different chunk splitting of the same media file, thus responding to different QOS requests.

\subsubsection{MULTIMEDIA STREAMING FROM CLOUD}

Fig1 Architectural design

\subsection{MODULE DESCRIPTION}

\subsubsection{USERINTERFACE DESIGN}

The goal of user interface design is to create the user's fundamental interaction as mere and efficient as possible, in terms of achieving user goals what is often called usercentered design. Graphic design may be utilized to support its usability. The design process must balance technical functionality and visual elements to create a system that is not only operational but also usable and adaptable to changing user needs.

\subsubsection{THE FIBONACCI VIDEO DIFFUSION APPROACH}

This module develop for QOS technique able to manage cloud resources in order to both guarantee service continuity and reduce the provisioning initial delay of the streaming multimedia files[3]. The chunks are used for improves the system performance, allowing the distributed and parallel execution of the tasks needed to the service provisioning. However, once the number of chunks is fixed, different performance can be observed concerned to the splitting strategies, which refer to the decisions about the size of each chunk. Splitting strategies influence different aspects of the system performance. The initial delay perceived by user, the service continuity, and. This process helps to overhead due to the parallel execution, in terms of system efficiency.
The Multimedia Streaming activity deals with user requests. Users request content with media list what file the user want to view or watch. The different chunks are then recovered and tailored, according to the requested characteristics, by a number of cloud server equal to the chunks number. In this module gather the video files from different cloud servers with in a network area and video chunks are stored in number of cloud servers. When client use our application list of videos will display in the user browsing window. The user can select any of the video from the window what they wish to watch, that particular file is send as a request to the resource manager that resource manager tailors the all chunks from different cloud servers and response to the client those who request the video.

\subsubsection{RESOURCE MANAGER MANAGEMENT}

Multimedia streaming services involve stringent QOS requirements, typical of soft real time applications. The resource manager of the CMS is in pursuit of fairly distributing the task load across server clusters, and hence, it is of importance and interest to be able to cope with load balancing in the CMS. Resource manager and a number of server clusters are coordinated by a cluster head[2]. Different from the decentralized CMS, each time it receives clients' requests for multimedia service tasks, the resource manager of the centralized CMS stores the global service task load information collected from server clusters, and decides the amount of client's requests assigned to each server cluster so that the load of each server cluster is distributed as balanced as possible in terms of the cost of transmitting multimedia data between server clusters and clients. 


\section{CONCLUSION AND FUTURE WORKS}

In the existing system client search for the particular server for an requested data or video to the where it take more time to process it and then only it was delivered to client from server. In order to avoid this problem videos are splitted by using this Fibonacci approach as they are divide into number of chunks. This chunks are present in all servers which will prevent loading buffering problem are reduced . It will lead to quick video or file display to the end user without any delay thus enabling the provisioning of services with different QOS levels.

\section{REFERENCES}

[1] W. Zhu, C. Luo, J. Wang, and S. Li, -Multimedia cloud computing:An emerging technology for providing multimedia services and applications, |IEEE Signal Process. Mag., vol. 28, no. 3, pp. 59-69, May 2011.

[2]Yung-Cheng Kao, Chung-Nan Lee, Peng-Jung Wu, and Hui- Hsiang Kao - - A Network Coding Equivalent Content Distribution Scheme for Efficient Peer-to-Peer Interactive VoD Streaming\| VOL. 23, NO. 6, JUNE 2012.

[3] W. Hui, H. Zhao, C. Lin, and Y. Yang,-Effective load balancing forcloud-based multimedia system, $\|$ in Proc. Int. Conf. Electron. Mech. Eng.Inform. Technol., 2011, pp. 165168.

[4]Dynamic Multiservice Load Balancing inCloud-Based Multimedia System Chun-Cheng Lin, Member, IEEE, HuiHsin Chin,Student Member, IEEE, and Der-Jiunn Deng, MARCH 2014.

[5] C.-F. Lai, Y.-M. Huang, and H.-C. Chao, "DLNA-based multimedia

IEEE Syst. J., vol. 4, no. 2, pp. 262-270, Jun. 2010.

sharing system over OSGI framework with extension to P2P network," 